\title{
The Effect of Nutrition Education on Nutrition Treatment of Chronic Kidney Disease
}

\author{
Lin Lian ${ }^{1}$, Zhong Xiaochun ${ }^{1}$, Huang Shaomin ${ }^{1}$, Zhang Xuemei ${ }^{1, *}$
}

${ }^{I}$ Department of Nephrology, Center of Nephrology and Urology, The Seventh Affiliated Hospital, Sun Yat-sen University, Shenzhen, China, 518107

*Corresponding author: Xuemei Zhang, https://orcid.org/0000-0003-1509-7485,Department of Nephrology, Center of Nephrology and Urology, The Seventh Affiliated Hospital, Sun Yat-sen University, 628 New Lake Street, Guangming New District, Shenzhen, China, 518107, Email: zhangxm23@mail.sysu.edu.cn

Article History Received 20 July 2020 Accepted 25 July 2020 Published 30 September 2020

Cite this Article Lin Lian, Zhong Xiaochun, Huang Shaomin, Zhang Xuemei. The Effect of Nutrition Education on Nutrition Treatment of Chronic Kidney Disease [J].Medical Research, 2020.2(3):20-24, http://dx.doi.org/10.6913/MRHK.202009_2(3).0004

Copyright (C) 2020 Creative Publishing Co., Limited. All rights reserved. Email:mrhk26640333@gmail.com.

ABSTRACT Chronic kidney disease (CKD) is a common chronic disease in the clinic. Patients are often at the risk of malnutrition due to impaired renal function [1-3]. In order to reduce the risk of malnutrition, it is advocated to implement nutritional therapy for patients with chronic kidney disease, but some patients have poor compliance and irregular dietary behavior, so effective intervention means are needed. Nutritional education is a specialized nutritional intervention measure. In order to assess the application effect of nutritional education in the nutritional therapy of chronic kidney disease, a randomized control trial was conducted on 100 patients with chronic kidney disease who received nutritional therapy in our hospital from August 2020 to October 2020.

Keywords effect of nutrition education; nutrition treatment; chronic kidney disease

\section{Materials and methods}

\section{1 general data of patients}

100 patients with chronic kidney disease receiving nutritional therapy in our hospital were selected as the research objects, and randomly divided into 50 patients per group from August 2020 to October 2020. Control group: 32 males and 18 females, aged from 40 to 84 years old, with an average of (62.13 \pm 10.34$)$ years old; Observation group: 33 males and 17 females, aged from 40 to 83 years old, with an average of (61.68 \pm 10.29$)$ years old. Comparison of age and sex between groups, $\mathrm{P}>0.05$. The study was approved by the Board of Medical Ethics and patients were given informed consent to the study.

\subsection{Methods}

Nutritional therapy: according to patients' quality to calculate daily intake of energy (about $30 \sim 35$ $\mathrm{kcal} / \mathrm{kg}$ ), fat intake accounted for $25 \%$ of total calories, protein intake are different in various stages of renal injury (mild renal damage stage daily intake of $0.8 \sim 1.0 \mathrm{~g} / \mathrm{kg}$, moderate renal impairment stage $0.6 \sim 0.7 \mathrm{~g} / \mathrm{kg}$, 
Lin Lian, Zhang Xuemei, et al. The Effect of Nutrition Education on Nutrition Treatment of Chronic Kidney Disease Medical Research ISSN 2664-0333 eISSN 2664-0341 Volume 2 Issue 3 http://dx.doi.org/10.6913/MRHK.202009_2(3).0004

severe kidney damage stage $0.5 \sim 0.6 \mathrm{~g} / \mathrm{kg}$, uremia phase $0.3 \sim 0.5 \mathrm{~g} / \mathrm{kg}$ ).

Both groups received initial nutritional education before starting nutritional therapy, briefly explained the considerations during nutritional therapy, and distributed nutritional therapy health handbook to patients.

Nutritional education was carried out by nutritionists in the department of Nutrition in the observation group during the nutritional therapy, while nutritional education was not carried out in the control group.

The Specific contents of nutritional education, 1) The restriction of water intake. during hemodialysis treatment, patients should control the daily weight gain rate within $5 \%$ as much as possible, and patients with excessive water intake easily lead to Oedema and weight gain, so we should strictly Limit water intake, and understand the water content in food, reducing the food with high water content. For an example, when a patient is thirsty, he or she can contain a sip water or chew gum. 2) The restriction in sodium intake. The daily sodium intake of the patient is strictly controlled, and the intake is determined based on whether the patient has high blood pressure. If the patient's blood pressure is normal, the daily salt intake should be 3 to $5 \mathrm{~g}$. If the patient has mild to moderate hypertension, the daily salt intake should be controlled within $2 \mathrm{~g}$. If the patient has severe hypertension, the daily salt intake should be controlled within $1 \mathrm{~g}$, and the consumption of preserved food should be avoided as much as possible. 3) The restriction in Potassium Intake. We need to explain the dangers of excessive potassium intake to patients and chart the potassium content of various foods. Especially need to pay attention to mushrooms, oranges, red dates and other high potassium content of food, and avoid eating high potassium food in the daily diet. When cooking dishes, appropriate measures can be taken to remove potassium, such as slicing and soaking vegetable rhizome parts before cooking, blanching vegetables and so on. 4) The limitation in phosphorus Intake. we need to explain to patients about the danger of excessive intake of phosphorus, and make a table of phosphorus content of various foods, especially the seafood, animal offal, egg yolk and other foods rich in phosphorus, which should be forbidden to eat.

\subsection{Observation index}

The scores of dietary knowledge awareness, dietary behavior management, the indexes of calcium and phosphorus metabolism (blood calcium, blood phosphorus), nutritional status (proalbumin, transferrin, albumin, hemoglobin), life quality score, duration of nutritional therapy, and hospitalization time were compared between the two groups.

The score of Nephrotic diet knowledge awareness. patients were investigated by using self-made Nephrotic diet knowledge awareness questionnaire on admission and discharge, the total score of the volume was 100, the higher the score, the more knowledge the patients know about Nephrotic diet.

The score of diet behavior management [4]. The diet compliance behavior scale of kidney disease was used to evaluate on admission and discharge, which was divided into five areas: self-care, water restriction, low-salt diet, low-potassium diet, and facing difficulties. The highest score in each area was 100, which was positively correlated with dietary behavior compliance.

Quality of Life score [5]. The World Health Organization Quality of Life Assessment (WHOQOL-BREF) was used to evaluate the quality of life on admission and discharge. The scale was divided into 4 fields, including physiology, psychology, environment and social relations. The score of each field was 0-100, and the score was directly proportional to the quality of life.

\subsection{The method of statistics}

$\chi 2$ test was performed on the enumeration data (n), $\mathrm{t}$ test was performed on the measurement data $(\bar{x} \pm s)$ by SPSS 26.0 , and $\mathrm{P}<0.05$ indicates that the difference is statistically significant.

\section{Result}

\subsection{The Comparison of knowledge on nephropathy}


The scores of dietary knowledge of nephropathy on discharge were higher in the two groups than on admission, while the scores of which on discharge were higher in the observation group than in the control group, $\mathrm{P}<0.05$.

Table 1 The awareness score on Nephropathy diet knowledge $(\bar{x} \pm s$, point $)$

\begin{tabular}{lcc}
\hline Group $(\mathbf{n}=\mathbf{5 0})$ & on admission & on discharge \\
\hline Control group & $60.56 \pm 12.27$ & $78.39 \pm 10.78^{\#}$ \\
observation group & $60.89 \pm 12.15$ & $87.76 \pm 9.54^{\# *}$ \\
\hline
\end{tabular}

Note: To compare with the group on admission, $\# \mathrm{P}<0.05$; To compare with the control group, ${ }^{*} \mathrm{p}<0.05$.

\subsection{The comparison of dietary behavior management scores}

The scores of dietary behavior management on discharge were higher in both groups than on admission, while those in observation group were higher than those in control group, $\mathrm{p}<0.05$.

Table 2. The comparison of dietary behavior management scores ( $\bar{x} \pm s$, point)

\begin{tabular}{|c|c|c|c|c|c|c|}
\hline $\begin{array}{l}\text { Group } \\
(n=50)\end{array}$ & time & self-care & $\begin{array}{c}\text { water } \\
\text { restriction }\end{array}$ & $\begin{array}{c}\text { low-salt } \\
\text { diet }\end{array}$ & $\begin{array}{c}\text { low-potassium } \\
\text { diet }\end{array}$ & $\begin{array}{c}\text { facing } \\
\text { difficulties }\end{array}$ \\
\hline Control & $\begin{array}{c}\text { on } \\
\text { admission }\end{array}$ & $\begin{array}{c}71.84 \pm 5.4 \\
6\end{array}$ & $72.19 \pm 6.02$ & $71.92 \pm 5.91$ & $71.35 \pm 5.48$ & $71.64 \pm 5.13$ \\
\hline group & $\begin{array}{c}\text { on } \\
\text { discharge }\end{array}$ & $\begin{array}{c}78.21 \pm 6.3 \\
5^{\#}\end{array}$ & $78.94 \pm 6.75^{\#}$ & $78.35 \pm 6.43^{\#}$ & $77.64 \pm 6.30^{\#}$ & $\begin{array}{c}77.90 \pm 6.29 \\
\#\end{array}$ \\
\hline observation & $\begin{array}{c}\text { on } \\
\text { admission }\end{array}$ & $\begin{array}{c}71.97 \pm 5.5 \\
2\end{array}$ & $72.32 \pm 6.14$ & $72.07 \pm 5.98$ & $71.47 \pm 5.56$ & $71.83 \pm 5.27$ \\
\hline group & $\begin{array}{c}\text { on } \\
\text { discharge }\end{array}$ & $\begin{array}{c}85.30 \pm 7.0 \\
9^{\# *}\end{array}$ & $\begin{array}{c}86.07 \pm 6.83^{\#} \\
*\end{array}$ & $\begin{array}{c}86.12 \pm 6.76^{\#} \\
*\end{array}$ & $85.09 \pm 6.45^{\# *}$ & $\begin{array}{c}85.78 \pm 6.92 \\
\# *\end{array}$ \\
\hline
\end{tabular}

Note: To compare with the group on admission, $\# \mathrm{P}<0.05$; To compare with the control group, ${ }^{*} \mathrm{p}<0.05$

\subsection{The Comparison in the indexes of calcium and phosphorus metabolism.}

The levels of serum calcium and phosphorus in the two groups were lower on discharge than on admission, while those in the observation group were lower than those in the control group, $\mathrm{P}<0.05$.

Table 3. The Comparison in the indexes of calcium and phosphorus metabolism.

\begin{tabular}{|c|c|c|c|}
\hline Group $(n=50)$ & Time & $\begin{array}{c}\text { Serum calcium } \\
(\mathrm{mmol} / \mathrm{L})\end{array}$ & $\begin{array}{l}\text { Serum phosphat } \\
\text { e (mmol/L) }\end{array}$ \\
\hline \multirow{2}{*}{ Control group } & on admission & $2.58 \pm 0.72$ & $2.34 \pm 0.24$ \\
\hline & on discharge & $1.76 \pm 0.39^{\#}$ & $2.10 \pm 0.19^{\#}$ \\
\hline observation & on admission & $2.53 \pm 0.75$ & $2.30 \pm 0.26$ \\
\hline group & on discharge & $1.37 \pm 0.34^{\# *}$ & $1.81 \pm 0.18^{\# *}$ \\
\hline
\end{tabular}

Note: To compare with the group on admission, $\# \mathrm{P}<0.05$; To compare with the control group, ${ }^{*} \mathrm{p}<0.05$.

\subsection{The comparison in Quality of life score.}

The quality of life scores in both groups were higher on discharge than on admission, while those in the observation group were higher on discharge than those in the control group, $\mathrm{P}<0.05$. 
Lin Lian, Zhang Xuemei, et al. The Effect of Nutrition Education on Nutrition Treatment of Chronic Kidney Disease Medical Research ISSN 2664-0333 eISSN 2664-0341 Volume 2 Issue 3 http://dx.doi.org/10.6913/MRHK.202009_2(3).0004

Table 5. The comparison in Quality of life score

\begin{tabular}{cccccc}
\hline $\begin{array}{c}\text { Group } \\
(\mathbf{n = 5 0 )}\end{array}$ & Time & $\begin{array}{c}\text { Physiologica } \\
\text { l field }\end{array}$ & $\begin{array}{c}\text { Psychological } \\
\text { field }\end{array}$ & $\begin{array}{c}\text { Environm } \\
\text { ental field }\end{array}$ & $\begin{array}{c}\text { social relation } \\
\text { shipfield }\end{array}$ \\
\hline & on admission & $74.56 \pm 5.09$ & $75.38 \pm 5.20$ & $\begin{array}{c}74.27 \pm 4.8 \\
1\end{array}$ & $75.09 \pm 5.18$ \\
Control group & & & & $81.35 \pm 5.0$ & $82.94 \pm 5.23^{\#}$ \\
& on discharge & $82.09 \pm 6.53^{\#}$ & $83.12 \pm 6.17^{\#}$ & $3^{\#}$ & \\
& & & & $74.39 \pm 4.7$ & $75.20 \pm 5.04$ \\
observation & on admission & $74.68 \pm 5.04$ & $75.52 \pm 5.13$ & 5 & \\
group & & & & $87.46 \pm 5.1$ & $88.57 \pm 5.69^{\# *}$ \\
& on discharge & $88.45 \pm 6.37^{\# *}$ & $89.34 \pm 6.28^{\# *}$ & $4^{\# *}$ & 8 \\
\hline
\end{tabular}

Note: To compare with the group on admission, $\# \mathrm{P}<0.05$; To compare with the control group, ${ }^{*} \mathrm{p}<0.05$.

\subsection{The Comparison of duration of nutritional treatment and hospital stay.}

Compared with the control group, the duration of nutritional treatment and hospital stay were significantly shortened in the observation group, $\mathrm{p}<0.05$.

Table 5. The Comparison of duration of nutritional treatment and hospital stay, $(\bar{x} \pm s, \mathbf{d})$.

\begin{tabular}{ccc}
\hline Group $(\mathbf{n = 5 0})$ & duration of Nutritional treatment & hospital stay \\
\hline Control group & $8.63 \pm 1.72$ & $17.45 \pm 3.96^{\#}$ \\
observation group & $6.81 \pm 1.40^{*}$ & $13.29 \pm 3.21^{\# *}$ \\
\hline
\end{tabular}

Note: Compared with the control group, $p<0.05$.

\section{Discussion}

Chronic kidney disease (CKD) is a kind of common chronic disease, which occurs all over the world. According to the survey, the probability of CKD among adults in the United States is about $13.1 \%$, and the prevalence rate of CKD among adults in most parts of China is about $8 \%$. This disease is one of the public health problems that endanger human health worldwide [6-8].

Clinically, maintenance hemodialysis is usually used in the treatment of chronic kidney disease, which can effectively remove the toxin molecules in the blood of patients and reduce the damaging effect of toxin on their renal function [9-11]. During maintenance hemodialysis treatment, patients with chronic kidney disease are at risk of malnutrition and prone to anemia. In order to reduce the risk of malnutrition in patients with chronic kidney disease, nutritional therapy is advocated clinically for patients with chronic kidney disease, and nutritional therapy has become the main treatment for chronic kidney disease [12-14].

However, the formulation of nutritional therapy diet for patients with chronic kidney disease is affected by many factors such as renal function, blood pressure, hemodialysis and so on. Low-salt diet often affects the color and flavor of food, resulting in the loss of appetite and poor dietary compliance among patients. Therefore, intervention measures are needed [15].

For this situation, our hospital carried out nutritional education and therapy for patients with chronic kidney disease (CKD), mainly explain nutritional knowledge for patients and guide the rational diet, which can improve the knowledge of kidney disease diet. what is more, this can make them fully understand the importance of nutritional therapy on kidney disease, and pay attention to diet, actively cooperate with nutritional therapy [16]. 
Lin Lian, Zhang Xuemei, et al. The Effect of Nutrition Education on Nutrition Treatment of Chronic Kidney Disease Medical Research ISSN 2664-0333 eISSN 2664-0341 Volume 2 Issue 3 http://dx.doi.org/10.6913/MRHK.202009_2(3).0004

Our study showed that: 1) The knowledge score of nephropathic diet and the score of diet behavior management were higher in the observation group than in the control group on discharge, $\mathrm{P}<0.05$, indicating that nutritional education can improve the nephrotic diet cognition of patients with chronic kidney disease, which can regulate their dietary behavior, and form a good dietary habit. 2) The levels of serum calcium and phosphorus in the observation group were lower than those in the control group, the levels of proalbumin, transferrin, albumin and hemoglobin in the observation group were higher than those in the control group on discharge, and the duration of nutritional treatment and hospital stay in the observation group were shorter than those in the control group, $\mathrm{P}<0.05$. This is mainly because nutritional education can improve the cognition of diet and compliance among patients, which can makesure that the implementation of nutrition treatment, thereby reducing the risk of malnutrition and correcting water- electrolyte disorders. 3) The quality of life scores in the observation group were higher than those in the control group on discharge, $\mathrm{P}<0.05$, this is mainly because nutritional education indirectly improves the nutritional status of patients, which is beneficial to reduce the impact of malnutrition on the quality of life of patients.

In conclusion, the implementation of nutritional education during nutritional treatment for patients with chronic kidney disease can effectively improve the dietary knowledge of kidney disease among patients, and is conducive to improving their compliance with dietary behavior management. Furthermore, the nutritional therapy can also improve the nutritional status and the metabolism of calcium and phosphorus, which would improve the quality of life and shorten the treatment time.

\section{Reference}

[1]Titze, Stephanie,Schmid, Matthias,Koettgen, Anna et al.Disease burden and risk profile in referred patients with moderate chronic kidney disease: composition of the German Chronic Kidney Disease (GCKD) cohort[J].Nephrology, dialysis, transplantation,2015,30(3):441-451.

[2]Chen, Teresa K.,Estrella, Michelle M.,Astor, Brad C. et al.Longitudinal changes in hematocrit in hypertensive chronic kidney disease: results from the African-American Study of Kidney Disease and Hypertension (AASK)[J].Nephrology,dialysis, transplantation,2015,30(8):1329-1335.

[3]Jayasekara, Kithsiri Bandara,Dissanayake, Dhammika Menike,Sivakanesan, Ramiah et al.Epidemiology of Chronic Kidney Disease, With Special Emphasis on Chronic Kidney Disease of Uncertain Etiology, in the North Central Region of Sri Lanka[J].Journal of epidemiology,2015,25(4):275-280.

[4]Jie Chen Xiaoping Lou,Hongxia.Shen etc.Effect of Retriever Diet Management on Dietary Compliance and Nutritional Status of Peritoneal Dialysis Patients[J]Chinese Journal of Nursing,2019,54(4):495-500.

[5]Yuantao Dou,Jiquan Fang.Introduction to the Chinese version of the World Health Organization Quality of Life Scale and its use[J] Modern rehabilitation,2000,4(8):1127-1129,1145.

[6]Jing, Jiaojiao,Kielstein, Jan T.,Schultheiss, Ulla T. et al.Prevalence and correlates of gout in a large cohort of patients with chronic kidney disease: the German Chronic Kidney Disease (GCKD) study[J].Nephrology, dialysis, transplantation,2015,30(4):613-621.

[7]Marius A Ovrehus,Petra Zurbig,Bjorn E Vikse Stein I Hallan et al.Urinary proteomics in chronic kidney disease: diagnosis and risk of progression beyond albuminuria[J].Clinical proteomics,2015,12(Jan./Jun.):21 108.

[8]Tobino, Kyoko,Muso, Eri,Iwasaki, Yukako et al.Gender- and disease-specific urinary thioredoxin in chronic 
kidney disease patients with or without type 2 diabetic nephropathy[J].Nephrology.,2015,20(5):368-374.

[9]Marcus, Robin L.,LaStayo, Paul C.,Ikizler, T. Alp et al.Low Physical Function in Maintenance Hemodialysis Patients Is Independent of Muscle Mass and Comorbidity[J].Journal of renal nutrition,2015,25(4):371-375.

[10]Tomayko, Emily J.,Kistler, Brandon M.,Fitschen, Peter J. et al.Intradialytic Protein Supplementation Reduces Inflammation and Improves Physical Function in Maintenance Hemodialysis Patients[J].Journal of renal nutrition,2015,25(3):276-283.

[11]Qiurong Nie,Yan Chen.Effects of Dietary Guidance on Self-Nutritional Management of Inpatients with Chronic Kidney Disease.Contemporary Nurses. 2020,27(1):19-21

[12]Yunfei Bao,Hong Wang,Chu Zhou etc.Investigation of nutritional status and diet of outpatients with integrated management of chronic kidney disease[J]Blood purification in China,2017,16(3):154-157.

[13]Falei Zheng.Nutritional treatment in patients with chronic kidney disease:well defined goals,Improved guidance[J]Journal of Kidney Disease and Dialysis Kidney Transplantation,2017,26(4):345-346.

[14]Feifei Jiang,Jie yao,Fei Jiang etc.Effect of nutritional intervention based on chronic kidney disease management system on nutritional status of patients with chronic kidney disease[J]General Medicine Clinical Education,2018,16(5):577-579.

[15]Yan Yang,Faxian Ding,Xiao Ma etc.Investigation and Analysis on Individualized Target Nutrition Management in Patients with Chronic Kidney Disease[J] Journal of Hebei Medical University,2017,38(5):525528,534 .

[16]Lixia Meng,Jun Zhao.Effect of Individualized Nursing and Nutritional Health Education on Nutritional Treatment of Chronic Kidney Disease[J]International Journal of Nursing,2019,38(24):4111-4113. 